\section{Genetic Diversity of Species of Chrysanthemum and Related Genera and Groundcover Cultivars Assessed by Amplified Fragment Length Polymorphic Markers}

\author{
Xuejuan Chen
}

Department of Ornamental Horticulture, School of Landscape Architecture, Beijing Forestry University, 35 Qinghua East Road, Beijing 100083, China

Ming Sun

Department of Ornamental Horticulture, School of Landscape Architecture, Beijing Forestry University, 35 Qinghua East Road, Beijing 100083, China; and the China National Engineering Research Center for Floriculture, Beijing 100083, China

\begin{abstract}
Jianguo Liang and Hui Xue
Department of Ornamental Horticulture, School of Landscape Architecture, Beijing Forestry University, 35 Qinghua East Road, Beijing 100083, China

Qixiang Zhang ${ }^{1}$

Department of Ornamental Horticulture, School of Landscape Architecture, Beijing Forestry University, 35 Qinghua East Road, Beijing 100083, China; and the China National Engineering Research Center for Floriculture, Beijing 100083, China
\end{abstract}

Additional index words. breeding, Chrysanthemum $\times$ morifolium, groundcover, hybridization, wild species

Abstract. Chrysanthemums have beautiful flowers with high ornamental value and rich
genetic diversity. Amplified fragment length polymorphism (AFLP) markers were used
to detect the relationships among 12 wild accessions and 62 groundcover chrysanthemum
cultivars. Nineteen EcoRI/MseI primer combinations revealed 452 informative poly-
morphic bands with a mean of 23.8 bands and $71.5 \%$ polymorphic rate per primer pair.
Jaccard's coefficient of similarity varied from 0.64 to 0.89 , indicating much genetic
variation in chrysanthemums. The 74 accessions were classified into two major groups by
unweighted pair group method with the arithmetic averages (UPGMA). The dendrogram
showed that AFLP variability was closely correlated with both geographic distribution and
traditional classification of the wild accessions. Among all accessions, genetic relationship
was the most relevant factor in AFLP-marker clustering, whereas petal type was also
informative. AFLP technology could be very efficient for discriminating species of
chrysanthemum and its related genera and reconstruct their genetic relatedness.

Chrysanthemum (Chrysanthemum spp.; Asteraceae, Anthemideae) is not only a traditional Chinese flower, but also one of the

\footnotetext{
Received for publication 28 Dec. 2012. Accepted for publication $27 \mathrm{Feb} .2013$.

This project was supported by the National Key Technology R\&D Program for the 12th Five-Year Plan (No. 2012BAD01B07) and the Fundamental Research Funds for the Central Universities (No. TD2011-27), China.

We thank H.E. Zhao for providing Dendranthema indicum, which was collected in Korea. We are also grateful to the staff in the China National Engineering Research Center for Floriculture for their assistance with collecting and maintaining plant materials.

${ }^{1}$ To whom reprint requests should be addressed; e-mail zqxbjfu@126.com.
}

most important cut flower, garden flower, and potted ornamental floricultural crops in the world. Although a native from China (Editorial Committee of the Flora of China, 1983), chrysanthemums have been associated with various international cultures and have been widely cultivated for centuries for their beauty, fragrance, edibility, and medicinal values. Huge numbers of genotypes have been created by cultivation and breeding over thousands of years (Anderson, 2006).

Chrysanthemums are among the most important and valuable fall-blooming plants for their diversity of flower shapes, colors, and forms. Chen et al. (1995) started to breed new garden chrysanthemums in 1961, hybridizing dwarf cultivars with wild species through mixed pollination or open pollination. After generations of hybridization and selection, groundcover chrysanthemums were developed, and many outstanding cultivars were introduced to urban landscapes (Chen et al., 1995, 2005; Wang and Chen, 1990). The distinguishing features of groundcover chrysanthemums are compact sizes, abundant flowers, long bloom duration, and rich colors. They are also highly drought-tolerant.

To breed desirable chrysanthemum cultivars, it is important to select appropriate parents that are beautiful and genetically diverse. In addition, in consideration of the selfincompatibility of most chrysanthemums, the crossability of parents is important for crossbreeding. Breeders would have much better success in creating new cultivars if they knew the genetic relatedness of the parents before initiating crosses (Saxena et al., 2010). Molecular technology has been widely used in analyzing genetic relationships and diversity. For example, random amplified polymorphic DNA (RAPD) markers were used to analyze 18 chrysanthemum cultivars (Chrysanthemum $\times$ grandiflora) (Qin et al., 2002). Miao et al. (2007) classified 85 popular chrysanthemum cultivars into six groups using intersimple sequence repeat analysis. In contrast, the AFLP technique is a much more highly polymorphic and more efficient method that has been widely used to detect genetic variation. Evolutionary relationships in the genus Chrysanthemum were analyzed by AFLPs (Zhou and Dai, 2002), and genetic variation in potted and garden chrysanthemum cultivars was also tested (Han et al., 2007; Liu et al., 2008; Wu et al., 2007). However, no reports of genetic variation between wild species and groundcover chrysanthemum cultivars have been published. This article reports the genetic relatedness of 12 wild species and 62 groundcover chrysanthemum cultivars as revealed through AFLP analyses.

\section{Materials and Methods}

Plant materials. A total of 74 accessions were assessed in this study, including eight wild species of Chrysanthemum, four wild species [Ajania pacifica (Nakai) K. Bremer \& Humphries, Crossostephium chinense (L.) Makino, Opisthopappus longilobus Shih, and $O$. taihangensis (Ling) C. Shih] closely related to Chrysanthemum, and 62 groundcover cultivars of Chrysanthemum $\times$ morifolium with high ornamental value (Table 1). Chrysanthemum $\times$ morifolium have multiple evolutionary origins (Dai et al., 1998; Dowrick, 1952). Previous work suggested that $C$. indicum might be its primary ancestor (Fukai et al., 2002) with additional genetic contributions from C. vestitum and C. lavandulifolium (Zhou and Dai, 2002). These three species, as well as $C$. chanetii and $C$. nankingense, were sampled as wild representatives of groundcover chrysanthemum ancestors. Ajania pacifica, Crossostephium chinense, Opisthopappus longilobus, and $O$. taihangensis were included because they have been frequently hybridized with Chrysanthemum in recent years. Chrysanthemum indicum was obtained from Korea in July 2009 and the other wild species were 


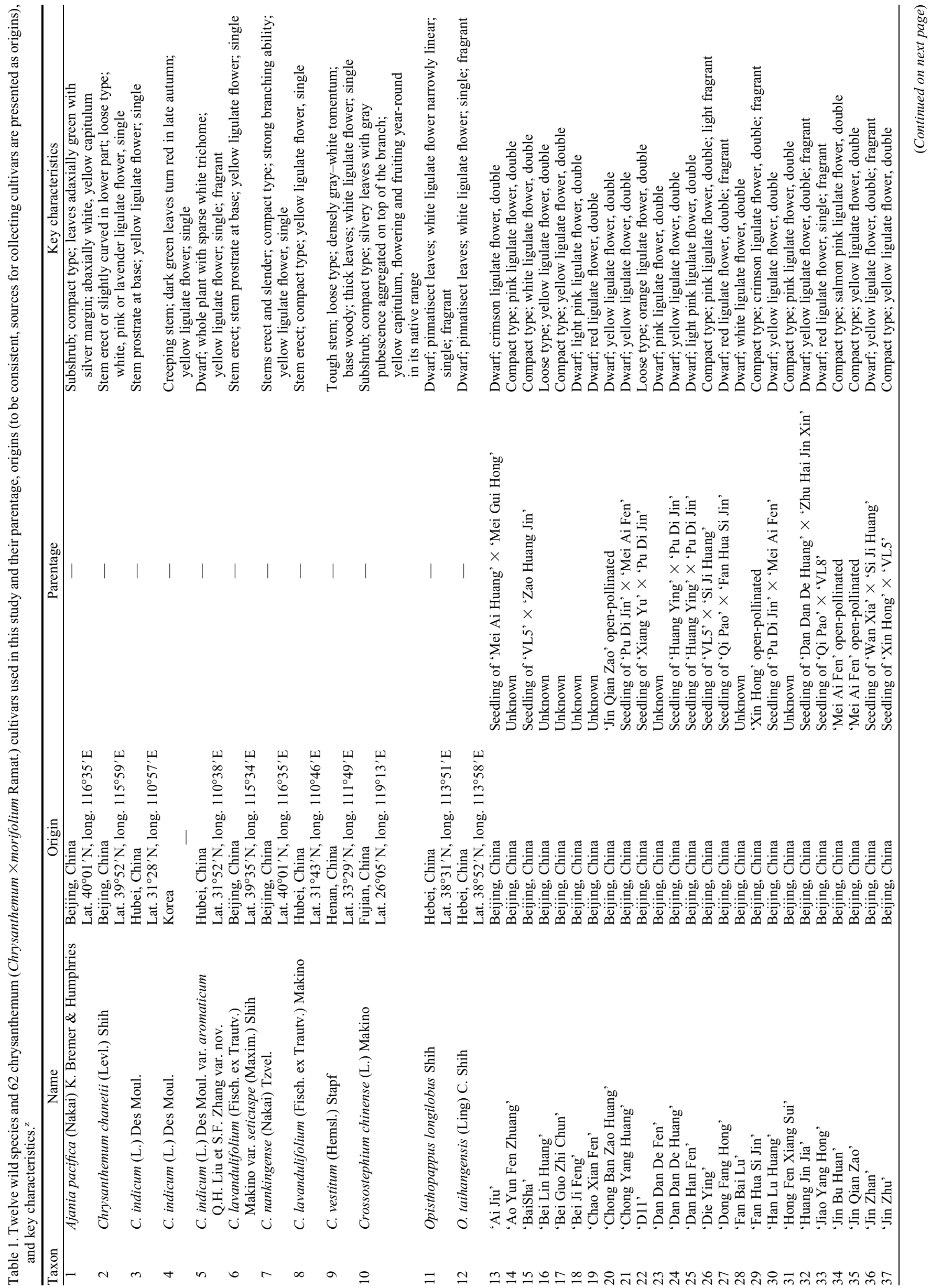




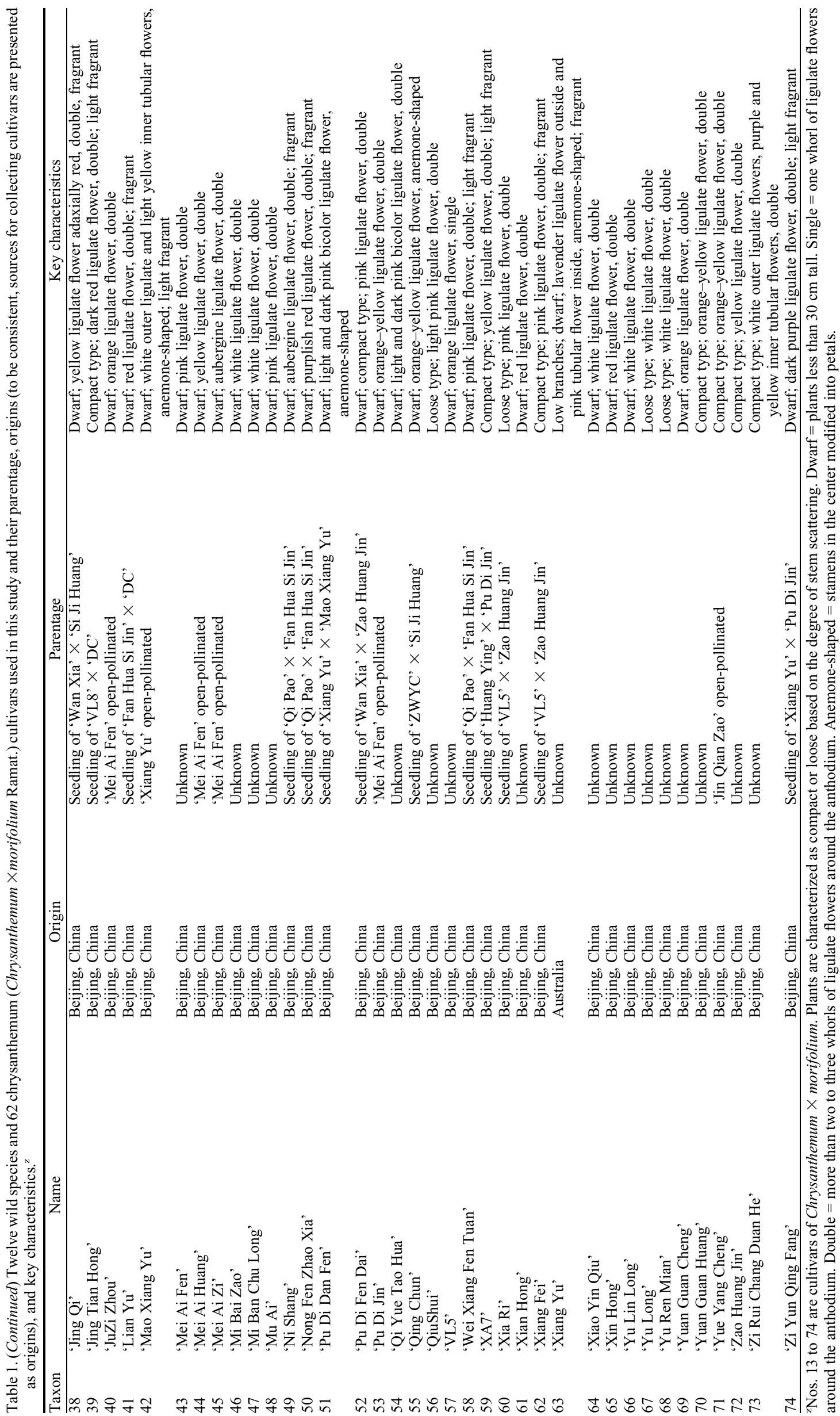


collected from several provinces in China between 2005 and 2010 (Table 1, Nos. 1 to 12). Groundcover cultivars in this study included 53 cultivars bred and selected in Beijing between 2004 and 2011 and nine groundcover cultivars introduced earlier (Table 1, Nos. 13 to 74). All of these taxa were grown in both greenhouses and fields at the China National Engineering Research Center for Floriculture (Beijing, China).

DNA extraction. Fresh young leaves were collected from greenhouse-grown plants of each accession and dried in silica gel desiccant. Approximately $0.40 \mathrm{~g}$ silica gel-dried leaves of each sample were weighed and ground to a fine powder in liquid nitrogen using a pestle and mortar. Genomic DNA was extracted from the powder using the DNA Secure Plant Kit (Tiangen Biotech, Beijing, China) according to the manufacturer's instructions. The quality and concentration of DNA were estimated with Smart Ladder (TaKaRa Biotechnology, Dalian, China) on a $1 \%$ agarose gel containing Gel Red (Biotium, OPE Technology Development, Shanghai, China) at $0.1 \mu \mathrm{g} \cdot \mathrm{mL}^{-1}$ in $1 \times$ Tris-acetateEDTA (TAE) buffer and visualized under the Gel Doc XR Imaging System (Bio-Rad, Shanghai, China). The DNA of each sample was adjusted to $50 \mathrm{ng} \cdot \mu \mathrm{L}^{-1}$ concentration and stored at $-20{ }^{\circ} \mathrm{C}$.

AFLP analysis. AFLP restriction, ligation, preselective amplification, and selective amplification reactions were conducted in a Techne TC-512 Thermal Cycler (Bibby Scientific, Staffordshire, U.K.). The analyses were performed as described by Vos et al. (1995) with modifications. Specifically, 100 ng DNA from each of the 74 samples was digested and ligated in a total volume of $20 \mu \mathrm{L}$ with $3 \mathrm{U}$ EcoRI (New England BioLabs, Beijing, China), $3 \mathrm{U}$ Mse I, $1.0 \mu \mathrm{L}$ of $1 \times \mathrm{T} 4$ DNA ligase buffer, $0.4 \mu \mathrm{L}$ of EcoRI adaptor (5 pmol), $0.4 \mu \mathrm{L}$ of $M s e \mathrm{I}$ adaptor (50 pmol), $0.4 \mu \mathrm{L}$ T4 DNA ligase, and double-distilled $\mathrm{H}_{2} \mathrm{O}$ $\left(\mathrm{ddH}_{2} \mathrm{O}\right)$ for $12 \mathrm{~h}$ at $37{ }^{\circ} \mathrm{C}$.

Polymerase chain reaction (PCR) primers adapter sequences are as follows: EcoRI adapter-5' -CTCGTAGACTGCGTACC-3', 3'-CTGACGCATGGTTAA-5' ; and $M s e$ I adapter: 5'-GACGATGAGTCCTGAG-3', 3'-TACTCAGGACTCAT-5' (Hill et al., 1996; Sun Ming, 2007). We used 280 pairs of EcoRI (E) and MseI (M) primers to analyze our 74 accessions. Finally, 19 high polymorphism EcoRI+ MseI primer combinations were used for this study (Table 2). Preselective amplification was performed with $2 \mu \mathrm{L}$ restriction/ligation mixture, $50 \mathrm{ng}$ primer $E c o R \mathrm{I}-$ 00, $50 \mathrm{ng}$ MseI-00, $10 \times$ PCR buffer, $1.5 \mathrm{~mm}$ $\mathrm{MgCl}_{2}, 0.1 \mathrm{~mm}$ each dNTP (Biomiga; Beijing Biodee Biological Technology, Beijing, China), 0.6 U Taq DNA polymerase (Promega, Beijing, China), and $\mathrm{ddH}_{2} \mathrm{O}$ in a $20 \mu \mathrm{L}$ reaction. The profile was set to 30 cycles of $94{ }^{\circ} \mathrm{C}$ for $30 \mathrm{~s}, 56^{\circ} \mathrm{C}$ for $30 \mathrm{~s}$, and $72^{\circ} \mathrm{C}$ for $60 \mathrm{~s}$ and a final extension step of $5 \mathrm{~min}$ at $72^{\circ} \mathrm{C}$.

Preamplification product DNA was diluted 10 -fold with $\mathrm{ddH}_{2} \mathrm{O}$ and then used as the template for the selective amplification. The selective amplification reaction was performed in a $20-\mu \mathrm{L}$ mixture containing $2 \mu \mathrm{L}$ of template, $40 \mathrm{ng}$ EcoRI-00 + NNN primer, $40 \mathrm{ng}$ Mse I-00 $+\mathrm{NNN}$ primer, $10 \times$ PCR buffer, $1.5 \mathrm{~mm} \mathrm{MgCl}_{2}, 0.1 \mathrm{~mm}$ of each dNTP, 0.6 U Taq DNA polymerase, and $\mathrm{ddH}_{2} \mathrm{O}$. Amplification was performed as follows: cycles 1 to $12: 94{ }^{\circ} \mathrm{C}, 30 \mathrm{~s} ; 65^{\circ} \mathrm{C}, 30 \mathrm{~s}$, $72{ }^{\circ} \mathrm{C}, 60 \mathrm{~s}$, with annealing temperature reduced by $0.7^{\circ} \mathrm{C}$ per cycle to $56^{\circ} \mathrm{C}$; cycles 13 to 42: $94{ }^{\circ} \mathrm{C}, 30 \mathrm{~s} ; 56^{\circ} \mathrm{C}, 30 \mathrm{~s} ; 72{ }^{\circ} \mathrm{C}, 60 \mathrm{~s}$; final extension: $72^{\circ} \mathrm{C}, 5 \mathrm{~min}$. Each sample was run in three replications to ensure the stability of the results. Amplified DNA products were stored at $4{ }^{\circ} \mathrm{C}$.

After the selective amplification, $8 \mu \mathrm{L}$ loading dye (98\% formamide, $10 \mathrm{~mm}$ EDTA, $1 \mathrm{mg} \cdot \mathrm{mL}^{-1}$ bromophenol blue, and xylene cyanol) was added to each product. The mixture was denatured at $95{ }^{\circ} \mathrm{C}$ for $10 \mathrm{~min}$ and placed immediately on ice to minimize renaturation. Each sample $(\approx 6$ to $7 \mu \mathrm{L}$ mixture) was loaded onto a $6 \%$ denaturing polyacrylamide gel that was pre-equilibrated with preheated $1 \times$ Tris-borate-EDTA (TBE) buffer. The gels were pre-run at $80 \mathrm{~W}$ for 30 to $40 \mathrm{~min}$, run for an additional 100 to 120 min, then silver-stained, and dried overnight. Finally, polymorphic fragments on the gels were scored on a light box.

Data analysis. Polymorphic AFLP loci were separated as bands of different molecular weight and scored by visual inspection as

present (1) or absent (0). There were 100-bp ladders on both sides of each gel as standards, and every polymorphic fragment was scored. Fragments smaller than $50 \mathrm{bp}$ and larger than $600 \mathrm{bp}$ were excluded from the analysis.

The similarity among plants was estimated with Jaccard's similarity coefficient. Cluster analyses of similarity matrices were achieved by using the UPGMA in NTSYS-pc Version 2.1q (Rohlf, 2000).

\section{Results}

AFLP polymorphisms. Most selective $\mathrm{E}+3 / \mathrm{M}+3$ primer combinations yielded too few polymorphic bands. In contrast, $\mathrm{E}+$ $2 / \mathrm{M}+2$ primers generated too many bands that overlapped and were difficult to distinguish. $\mathrm{E}+3 / \mathrm{M}+2$ and $\mathrm{E}+2 / \mathrm{M}+3$ combinations produced abundant amplification products with sufficient polymorphism. Ultimately, 19 primer combinations were selected to examine the 74 accessions of chrysanthemum.

The 19 primer pairs produced a total of 452 AFLP bands across all plant samples (Table 3). There was wide variation in the number of polymorphic loci per primer pair, ranging from nine to 40 and averaging 23.8. Allele size ranged from 50 to $583 \mathrm{bp}$, which covered an adequate amount of the whole genome. The mean polymorphism rate was $71.5 \%$ with a range of $57.8 \%$ to $88.4 \%$. The

Table 2. Sequences of oligonucleotide adaptors and primers used for amplified fragment length polymorphism analysis.

\begin{tabular}{|c|c|c|}
\hline & Name & Sequence \\
\hline \multirow[t]{4}{*}{ Adaptor } & EcoRI & $5^{\prime}$-CTCGTAGACTGCGTACC-3' \\
\hline & & 3'-CTGACGCATGGTTAA-5' \\
\hline & MseI & 5'-GACGATGAGTCCTGAG-3' \\
\hline & & 3'-TACTCAGGACTCAT-5' \\
\hline \multirow[t]{4}{*}{ Primer } & EcoRI-00 & 5'-GACTGCGTACCAATTC-3' \\
\hline & MseI-00 & 5'-GATGAGTCCTGAGTAA-3' \\
\hline & $E c o R \mathrm{I}+\mathrm{NNN}{ }^{\mathrm{z}}$ & $5^{\prime}$-GACTGCGTACCAATTC+NNN-3' \\
\hline & $M s e \mathrm{I}+\mathrm{NNN}$ & $5^{\prime}$-GATGAGTCCTGAGTAA+NNN-3' \\
\hline
\end{tabular}

${ }^{\mathrm{z}} \mathrm{NNN}$ represents one to three random nucleotides.

Table 3. Levels of polymorphism, allele size, and polymorphism rate revealed by $19 \mathrm{EcoRI} / \mathrm{Mse} \mathrm{I}$ primer combinations for amplified fragment length polymorphism analyses of chrysanthemum species and cultivars.

\begin{tabular}{lccc}
\hline Primer combination & $\begin{array}{c}\text { Number of } \\
\text { polymorphic loci }\end{array}$ & $\begin{array}{c}\text { Allele size } \\
\text { range }(\mathrm{bp})\end{array}$ & $\begin{array}{c}\text { Polymorphism } \\
\text { rate }(\%)\end{array}$ \\
\hline E-AAG/M-CTG & 9 & $57-360$ & 62.3 \\
E-ACA/M-CAG & 27 & $62-413$ & 65.4 \\
E-ACC/M-CTG & 24 & $54-396$ & 73.9 \\
E-ACG/M-CTG & 23 & $55-427$ & 86.7 \\
E-ATA/M-CAC & 16 & $84-496$ & 66.3 \\
E-ATA/M-CCC & 12 & $56-456$ & 87.5 \\
E-ATA/M-CTG & 14 & $54-306$ & 79.1 \\
E-ACA/M-CG & 40 & $55-343$ & 75.5 \\
E-AGG/M-CG & 19 & $69-458$ & 74.7 \\
E-ATG/M-CG & 28 & $74-428$ & 79.1 \\
E-ATT/M-CG & 19 & $55-382$ & 70.6 \\
E-CA/M-CTG & 30 & $59-376$ & 81.7 \\
E-CG/M-CAG & 19 & $66-564$ & 75.2 \\
E-CT/M-CAG & 26 & $69-564$ & 66.7 \\
E-GT/M-CTG & 24 & $50-361$ & 70.1 \\
E-TA/M-CAG & 40 & $71-583$ & 88.4 \\
E-TT/M-CAG & 32 & $57-501$ & 80.5 \\
E-TT/M-CTG & 34 & $50-580$ & 76.4 \\
E-CC/M-CG & 16 & $50-457$ & 57.8 \\
Mean & 23.8 & $50-600$ & 71.5 \\
Total & 452 & & - \\
\hline
\end{tabular}


primer combination E-TA/M-CAG had the highest polymorphism rate $(88.4 \%)$ followed by E-ATA/M-CCC (87.5\%) and E-ACG/ M-CTG (86.7\%). The lowest polymorphism rate was observed with primer combination E-CC/M-CG (57.8\%), whereas E-AAG/ M-CTG and E-ACA/M-CAG produced only $62.3 \%$ and $65.4 \%$ polymorphic bands, respectively.

Genetic diversity and cluster analysis within 12 wild accessions. Jaccard's genetic similarity coefficients, calculated between accessions, varied from 0.66 to 0.82 , and UPGMA cluster analysis resolved the genetic relationships among the 12 wild accessions (Fig. 1). The dendrogram divided the species into two main clusters split at a similarity of 0.66 . Cluster A contained only one wild species from Fujian, Crossostephium chinense, whereas cluster $\mathrm{B}$ included the other 11 wild accessions collected in Korea and other provinces of China. Cluster B was further divided into six subclusters, from BI to BVI, at a similarity of 0.76 . Furthermore, subcluster BI consisted of two subgroups, BI-I with $C$. indicum (two accessions) and C. indicum var. aromaticum and BI-II with Opisthopappus longilobus and O. taihangensis. Chrysanthemum lavandulifolium var. seticuspe and $C$. nankingense were grouped into subcluster BIV. The other four taxa, A. pacifica,
C. chanetii, C. lavandulifolium, and $C$. vestitum, each comprised a monotypic subcluster.

The 12 accessions had different polymorphic fragments, indicating that the AFLP primer combinations had great discriminatory power and that the 12 wild species were highly variable. The dendrogram indicates that wild accessions were divided into different groups in accordance with both the geographic distributions and traditional classification.

Genetic diversity and cluster analysis within species and cultivars. As shown in Figure 2, Jaccard's genetic similarity coefficient ranged from 0.64 to 0.89 . All 74 accessions were divided into two clusters by UPGMA analysis. Cluster B contained only 'Zi Rui Chang Duan He', which appeared to be quite isolated from other accessions.

Cluster A contained the other 73 accessions and was divided into subclusters AI and AII at a similarity of 0.67 . Subcluster AII contained only 'Jin Qian Zao', an old cultivar. Subcluster AI contained all 53 groundcover cultivars and 12 wild species, indicating close relationships among them. This subcluster was further divided into groups AI-I and AI-II at a similarity of 0.68 . 'Han Lu Huang' and 'Chong Yang Huang' were the most closely related accessions in this study and formed a small subgroup (subgroup 2 in Fig. 2) with 'Mei Ai Zi' and 'Pu Di Jin'; these
Beijing descendants of 'Mei Ai Fen' shared dwarf phenotypes with yellow, orange-yellow, or aubergine double flowers. There were four additional subgroups of closely related cultivars that shared parents and petal type. Subgroup 1 comprised 'XA7', 'Dan Dan De Huang', and 'Dan Han Fen', all dwarf or compact offspring of 'Huang Ying' $\times$ 'Pu Di Jin' with ligulate, double flowers. 'Xiang Yu' was the female parent of 'Mao Xiang Yu' and 'Pu Di Dan Fen', and these three cultivars with bicolor, anemone-shaped flowers formed subgroup 3. 'Jing Qi' and 'Jin Zhan', both descendants of 'Wan Xia' $\times$ 'Si Ji Huang', formed subgroup 4 with fragrant yellow, ligulate, double flowers. In subgroup 5, 'Zao Huang Jin' was the male parent of 'Pu Di Fen Dai'. Finally, both 'Jing Tian Hong' and 'Lian Yu' (subgroup 6) had 'DC' as the male parent; both have red ligulate double flowers. In addition, all 53 groundcover chrysanthemums were grouped with 12 wild species in subcluster AI, indicating a close relationship among them.

\section{Discussion}

Compared with traditional morphological identification, molecular markers are much more effective and reliable for analyzing genetic relationships and diversity. Morphological

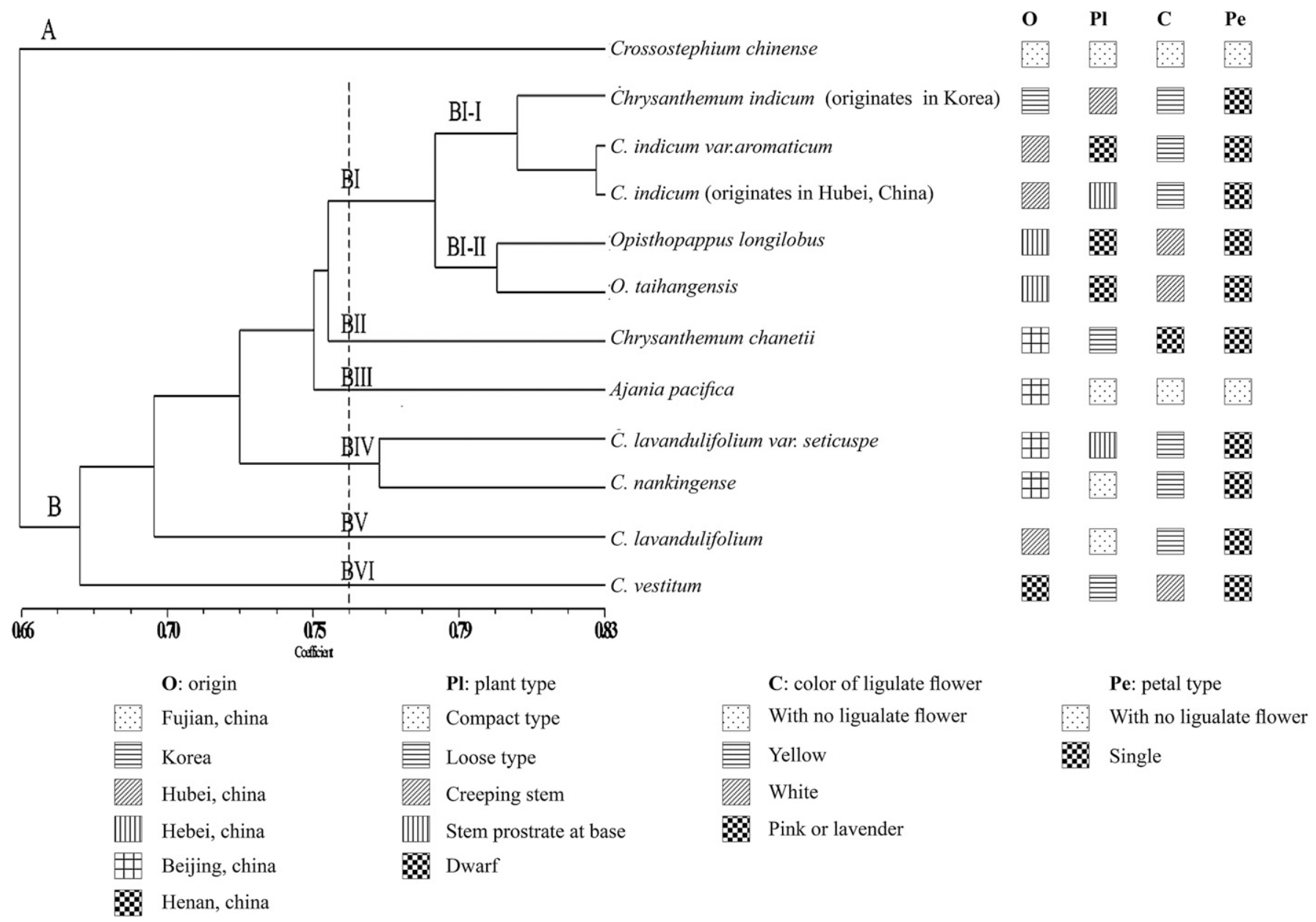

Fig. 1. Cluster analysis of eight wild Chrysanthemum accessions and four close relatives based on the matrix of genetic similarity of amplified fragment length polymorphism markers. 


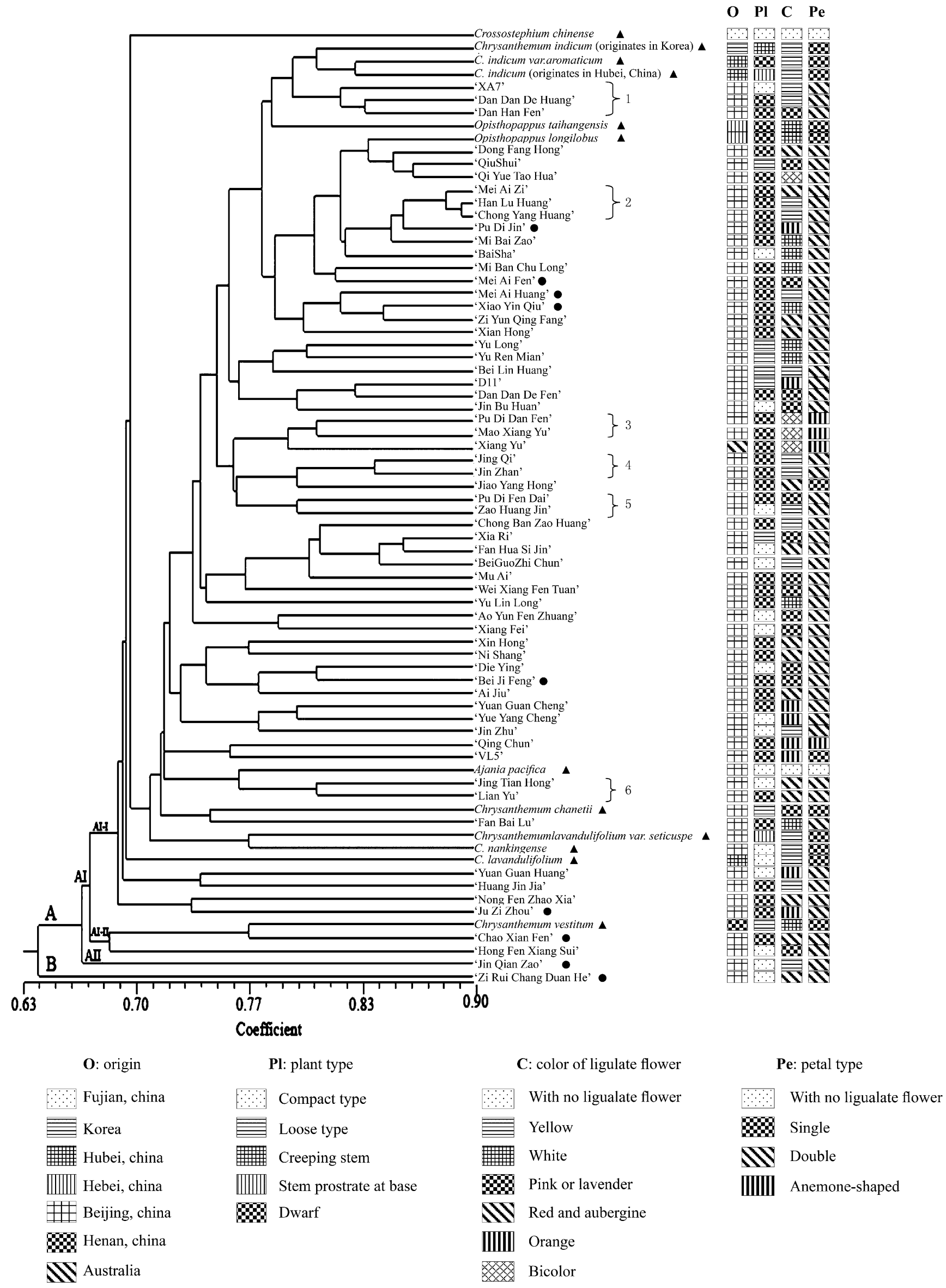

Fig. 2. Cluster analysis of 12 wild accessions and 62 Chrysanthemum $\times$ morifolium cultivars based on the matrix of genetic similarity of amplified fragment length polymorphism markers. $\mathbf{\Delta}=$ wild species; $\bullet=$ older cultivars of groundcover Chrysanthemum. 
identification is based on physical traits such as plant height, petal color, and fruit size that are usually vulnerable to external environmental effects. In contrast, molecular markers based on organismal DNA or proteins offer more sensitivity and efficiency (Lamote et al., 2002). Yuan et al. (2000) compared four molecular marker technologies, restriction fragment length polymorphisms, simple sequence repeats, AFLPs, and RAPDs, to study genetic diversity among maize inbred lines and concluded that AFLPs could detect the most differences at the DNA level.

In this study, AFLP markers were used to evaluate the genetic relationships among wild species and cultivars of chrysanthemum. The results supported the conclusions of previous studies that the AFLPs could distinguish species and cultivars effectively, even when they had high similarity indices. The primer pairs used in this study had an average $71.5 \%$ polymorphic rate, whereas in a RAPD study of chrysanthemums by Dai et al. (1998), the markers had only $15 \%$ polymorphism. Other studies have also concluded that AFLPs were a powerful tool for revealing genetic relationship in chrysanthemum cultivars (Han et al., 2007; Zhou and Dai, 2002). Obviously, the AFLP technique was an informative method for assaying genetic diversity, genetic relationships, and cultivar identity in chrysanthemums in this study.

The range of Jaccard's similarity coefficients obtained from the AFLP data indicated that there was significant genetic diversity between chrysanthemum species and cultivars. It also indicated that there existed wide genetic variation among groundcover chrysanthemums. In this study, 62 groundcover chrysanthemums were analyzed, $\approx 30 \%$ of this group of cultivars. Ortiz et al. (1998) suggested that a core collection of $10 \%$ of the available Musa germplasm stored in vitro may contain most genetic diversity; our core collection represented a much larger fraction of the total germplasm.

The AFLP markers used in this study could clearly differentiate the wild species by geographic region and traditional taxonomic delineation. For example, the three accessions of $C$. indicum clustered together, and the two accessions from Hubei, China, were more closely related. The two species of Opisthopappus also grouped together in the dendrogram of wild species. These results were consistent with previous reports (Wu et al., 2007; Zhou and Dai, 2002; Zhu et al., 2011). However, the two accessions of C. lavandulifolium did not group together.

When AFLP data from the groundcover accessions were analyzed along with the wild species, the resulting dendrogram indicated that most accessions were closely related both to other cultivars and to wild species. The older cultivars were the more closely related to wild species than were most recently bred groundcover cultivars. This finding was probably the result of the work of breeders, who created the oldest accessions by crossing cultivars with wild species, whereas the newer cultivars were mainly developed from older ones. The origin of C. morifolium remains controversial; however, the species is generally believed to be a hybrid complex derived from chance hybridization that occurred naturally among C. vestitum, C. indicum, C. lavandulifolium, and C. zawadskii Herbich (Chen et al., 1998; Dai et al., 1998; Wang et al., 2004). Therefore, the older cultivars were closely related to these wild parents.

The dendrogram also indicated that rich genetic diversity exists in chrysanthemums. After generations of intraspecific hybridization, the group of groundcover chrysanthemums are highly heterogeneous and have diverse genetic background. Furthermore, more distant hybridization with wild species can broaden the genetic base of chrysanthemum cultivars. Consequently, AFLP markers can provide valuable molecular data for investigating genetic relationships among closely related groups to guide future crosses, although traditional methods of differentiating varieties based on phenotypic characters should also be considered.

The results of this study were generally similar to those obtained by Miu et al. (2007) and $\mathrm{Wu}$ et al. (2007), which showed that genetic relationships within chrysanthemum were partly indicated by their petal types. The majority of cultivars had double flowers, but the accessions that shared anemoneshaped petals, 'Pu Di Dan Fen', 'Mao Xiang $\mathrm{Yu}$ ', and 'Xiang Yu', clustered together. Although flower color varies in chrysanthemums, there was no significant correlation between flower color and genetic as indicated by molecular markers. The relationships between the other morphological characters such as ligulate flower length, plant height, and flower diameter, and molecular markers need further testing and verification.

China is the center of chrysanthemum diversity with rich native germplasm resources. There are many species with desirable characteristics such as prolonged florescence and resistance to drought, salinity, and disease. These species have excellent potential to widen the genetic base for breeding new cultivars. Generally, parents that are more genetically distant would provide more variation. However, genetic improvement of chrysanthemums is hampered by its genome complexity, high level of heterozygosity, and the occurrence of both inbreeding depression and self-incompatibility (Anderson and Ascher, 2000; Li et al., 2007; Xu et al., 2009). Understanding genetic relationships and diversity in chrysanthemum is critical. Our AFLP data will help breeders to choose appropriate parents while breeding new varieties.

In conclusion, this research showed that the AFLP technique is an efficient method for revealing genetic relationships and variation among chrysanthemum cultivars. Furthermore, the results from this study will be useful for formulating effective breeding strategies and characterizing germplasm. These results form the foundation for further analysis that will include more wild species to cover more chrysanthemum genetic diversity.

\section{Literature Cited}

Anderson, N.O. 2006. Flower breeding and genetics: Issues, challenges and opportunities for the $21^{\text {st }}$ century. Springer, Dordrecht, The Netherlands.

Anderson, N.O. and P.D. Ascher. 2000. Fertility changes in inbred families of self-incompatible chrysanthemums (Dendranthema grandiflora). J. Amer. Soc. Hort. Sci. 125:619-625.

Chen, F., P. Chen, W. Fang, and H. Li. 1998. Cytogenetics of $F_{1}$-hybrids between two smallheaded cultivars of Dendranthema $\times$ grandiflorum and two wild Dendranthema species. Acta Hort. Sin. 25(3):308-309.

Chen, F., W. Fang, H. Zhang, Z. Guan, and G. Xu. 2005. New varieties of Chrysanthemum groundcover varieties. Acta Hort. Sin. 32:1167.

Chen, J., S. Wang, X. Wang, and P. Wang. 1995. Thirty years' studies on breeding ground-cover Chrysanthemum new cultivars. Acta Hort. 404:30-36.

Dai, S., J. Chen, and W. Li. 1998. Application of RAPD analysis in the study on the origin of Chinese cultivated Chrysanthemum. Acta Hort. Sin. 40:1053-1059.

Dowrick, G.J. 1952. The chromosomes of chrysanthemum, I: The species. Heredity 6:365-375.

Editorial Committee of the Flora of China. 1983 Flora of China. Science Press, Beijing, China.

Fukai, S., Y. Kamigaichi, N. Yamazaki, W. Zhang, and M. Gol. 2002. Distribution, morphological variations and cpDNA PCR-RFLP analysis of Dendranthema yoshinaganthum. J. Japan. Soc. Hort. Sci. 71:114-122.

Han, J., N. Hu, Y. Li, and F. Shang. 2007. Genetic diversity of chrysanthemum cultivars revealed by AFLP analysis. Acta Hort. Sin. 34:1041-1046.

Hill, M., H. Witsenboer, M. Zabeau, P. Vos, R. Kesseli, and R. Michelmore. 1996. PCR-based fingerprinting using AFLPs as a tool for studying genetic relationships in Lactuca spp. Theor. Appl. Genet. 93:1202-1210.

Lamote, V., I. Roldan-Ruiz, E. Coar, M.D. Loose, and E.V. Bockstaele. 2002. A study of genetic variation in Iris pseudoacorus populations using amplified fragment length polymorphisms (AFLPs). Aquat. Bot. 73:19-31.

Li, X., F. Chen, and H. Zhao. 2007. Compatibility of interspecific cross in Dendranthema genus. Acta Hort. Sin. 35:257-262.

Liu, M., S. Zhang, H. Liang, and Z. Zhen. 2008. AFLP analysis on the genetic diversity of some Chrysanthemum species. J. Agr. Univ. Hebei 31:48-52.

Miao, H., F. Chen, and H. Zhao. 2007. Genetic relationship of 85 Chrysanthemum [Dendranthema $\times$ grandiflora (Ramat.) Kiramura] cultivars revealed by ISSR analysis. Acta Hort. Sin. 34:1243-1248.

Ortiz, R., S. Madsen, and D. Vuylsteke. 1998. Classification of African plantain landraces and banana cultivars using a phenotypic distance index of quantitative descriptors. Theor. Appl. Genet. 96:904-911.

Qin, H., J. You, and J. Gan. 2002. RAPD analysis of 18 Chrysanthemum cultivars. Acta Hort. Sin 29:488-490.

Rohlf, F.J. 2000. NTSYS-pc: Numerical taxonomy and multivariate analysis system. Exeter Software, New York, NY.

Saxena, R.K., K.B. Saxena, R.V. Kumar, D.A Hoisington, and R.K. Varshney. 2010. Simple sequence repeat-based diversity in elite pigeonpea genotypes for developing mapping populations to map resistance to Fusarium wilt and sterility mosaic disease. Plant Breed. 129:135141.

Sun Ming. 2007. Studies on genetic relationships of ground-cover Chrysanthemum (Chrysanthemum 
morifolium Ramat.) and relative germplasm and breeding of new cultivars. Doctoral dissertation of Beijing For. Univ. p. 31.

Vos, P., R. Hogers, M. Bleeker, M. Reijans, T vande Lee, M Hornes, and M Zabeau. 1995. AFLP: A new technique for DNA fingerprinting. Nucleic Acids Res. 23:4407-4414.

Wang, C., J. Chen, and M.A. Jongsma. 2004 Molecular evolution and phylogeny of florist's chrysanthemum and related species. J. Beijing For. Univ. 26:91-96.
Wang, P. and J. Chen. 1990. Studies on breeding new cultivars of ground-cover chrysanthemum. Acta Hort. Sin. 17:223-224.

Wu, Z., H. Li, J. Liu, Z. Zuo, and R.C. Tian. 2007. Analyses of genetic diversity among 65 Chrysanthemum cultivars based on AFLP. J. Nanjing For. Univ. 31:67-70 (Natural Sciences).

$\mathrm{Xu}, \mathrm{Y}$., F. Chen, N. Teng, S. Chen, and L. Zhai. 2009. Identify the self-compatibility and change of protective enzyme activity in different styles of Chrysanthemum. Acta Hort. Sin. 36:59-64.
Yuan, L., J. Fu, X. Li, S. Zhang, X. Liu, Z. Peng, and L. Li. 2000. Comparison of genetic diversity among maize inbred lines based on RFLPs, SSRs, AFLPs and RAPDs. Acta Genet. Sin. 27:725-733.

Zhou, C. and S. Dai. 2002. AFLP analysis of some Dendranthema spp. J. Beijing For. Univ. 24:71-75.

Zhu, R., Y. Gao, L. Xu, and Q. Zhang. 2011. Genetic diversity of Aquilegia (Ranunculaceae) species and cultivars assessed by AFLPs. Genet. Mol. Res. 10:817. 